Research Article

\title{
Application of Artificial Intelligence for the Estimation of Concrete and Reinforcement Consumption in the Construction of Integral Bridges
}

\author{
Željka Beljkaš $\left(i,{ }^{1}\right.$ Miloš Knežević, ${ }^{1}$ Snežana Rutešić, ${ }^{1}$ and Nenad Ivanišević ${ }^{2}$ \\ ${ }^{1}$ Faculty of Civil Engineering, University of Montenegro, Podgorica 81000, Montenegro \\ ${ }^{2}$ Faculty of Civil Engineering, University in Belgrade, Belgrade 11120, Serbia \\ Correspondence should be addressed to Željka Beljkaš; zeljkab@ucg.ac.me
}

Received 8 February 2020; Revised 9 May 2020; Accepted 21 May 2020; Published 8 June 2020

Academic Editor: Carles Serrat

Copyright (C) 2020 Željka Beljkaš et al. This is an open access article distributed under the Creative Commons Attribution License, which permits unrestricted use, distribution, and reproduction in any medium, provided the original work is properly cited.

Estimation of basic material consumption in civil engineering is very important in the initial phases of project implementation. Its importance is reflected in the impact of material quantities on forming the prices of individual positions, hence on forming the total cost of construction. The construction companies use the estimate of material quantity, among other things, as a base to make a bid on the market. The precision of the offer, taking into account the overall conditions of the business realization, directly influences the profit that the company can make on a specific project. In the early stages of project implementation, there are not enough available data, especially when it comes to the data needed to estimate material consumption, and therefore, the accuracy of material consumption estimation in the early stages of project realization is smaller. The paper presents the research on the use of artificial intelligence for the estimation of concrete and reinforcement consumption and the selection of optimal models for estimation. The estimation model was developed by using artificial neural networks. The best artificial neural network model showed high accuracy in material consumption estimation expressed as the mean absolute percentage error, $8.56 \%$ for concrete consumption estimate and $17.31 \%$ for reinforcement consumption estimate.

\section{Introduction}

Cost estimation in construction represents a quantitative estimate of the probable resource expenses required for completing the activity [1]. A lot of factors affect the cost price. Each of these factors must be analysed, quantified, and estimated.

Estimating the final price requires a large number of elements to be synchronized. The process of defining the elements determining total costs includes the calculation of work quantities and then transferring them into expected costs. The basic elements or resources used and involved in the project during construction can be divided into several groups as follows:
(i) Work
(ii) Material
(iii) Equipment

(iv) Profit

(v) Time [2]

In order to carry out a project, it is necessary to organize teams with a large number of people. The initiative for entering the process of project implementation is started by the investor. The other participants in the project are a consultant, designer, expert supervision, contractor, and stakeholders.

In addition to the investor, the contractor has an important role on the project, since he represents a direct executor of the construction. An investor chooses a contractor based on certain criteria. Most commonly, these criteria require the construction of the highest quality structure with the minimum amount of money expended and in the shortest time possible. Clearly, these are the criteria that strive to idealize the entire course of the project and are therefore difficult to achieve. 
The investor and contractor estimate costs for themselves, separately. Depending on the cost estimation results, the decisions are made about the further steps in the project. It is often the case that certain project implementation is withdrawn after the estimation, or the design is significantly modified.

A large number of factors, such as availability, quality, and the level of details in technical documentation, estimation method as well as the expertise of people performing the estimate, determine its quality and reliability from the aspect of satisfactory accuracy. The initial phases of project implementation are characterized by the insufficient data quantity. Each subsequent phase brings new data. The availability of the necessary data helps to increase the accuracy of the estimate. In 1974, Barnes presented the dependence of cost estimate accuracy and the phase of the project (see Figure 1).

The price offered by a contractor for project implementation is usually the main and quite often the only criteria based on which the investor chooses a contractor. Thus, the procedure for choosing a contractor according to this criterion is extremely simple, since the bidder with the lowest price will be chosen as the contractor. The problem with this method of contractor selection is the inability to see whether the project will be successfully completed, i.e., whether the contractor who offered the lowest price will be able to complete the project in the expected or at least satisfactory way (taking into account expenses, quality, and time).

A preliminary project cost estimate is the first serious estimate to be made on a project. During the initial phases of project implementation, it is not necessary to have a sufficiently accurate cost estimate. Since the material is one of the elements that affects the overall cost of the project, in order to reach its estimate, it is necessary, among other things, to determine the quantities of construction material required in the project. After determining the quantities, they are multiplied by the corresponding unit prices of these materials, and thus, we arrive at an estimate of the material costs which is one of the items in the sum of total expenses. The advantage of the cost estimation algorithm, in which there is a cost breakdown of items, is that it updates the cost separately, position by position, when new data become available. Also, the positive side of this approach is that positions can be monitored separately, allowing decision makers to make better decisions about the project during its initial phase.

In this study, the estimate of material consumption is performed for the materials which are most present in bridge construction, reinforcement, and concrete. The estimate is based on the values taken from the bills of quantities and cost estimates from the design documentation based on which the works were contracted.

\section{Application of Artificial Neural Networks in Construction}

Application of neural networks, one of the artificial intelligence techniques, in construction engineering, is quite widespread. They can be used in all the project implementation phases. The journal Microcomputers in Civil Engineering published a paper in 1989 which refers to the use of neural networks in this area. The authors of this paper are Adeli and Yeh [4]. The application of neural networks in construction is becoming more and more common because, in addition to the wide range of abilities they have, the rapid development of software packages has contributed to this.

Neural networks can be used for different types of estimates. One of them is the cost estimation of different types of construction and it has been processed by a large number of authors in their works [5-11]. In addition to cost estimation, neural networks can be used to estimate the duration of construction projects, which was also a topic dealt with by certain authors [8, 12]. Estimation of material consumption for the facility construction is another one of the estimates that is possible to be performed by applying neural networks. Despite this, there are only few papers in the literature that present the results of neural network applications for the purpose of estimating material consumption.

Fragkakis et al. represented the conceptual model for cost estimation of bridge foundations, which also gives the estimation of material consumption. Independent variables, which are relevant to the model, were identified by experts in the interviews. For defining this model, the authors used the stepwise regression methodology in order to determine whether the results were consistent with the expert opinion. The main assumptions underlying the correct application of the regression method were examined, and the necessary adjustments were made. The proposed method of conceptual cost estimation and material consumption estimation provides quick and reliable results that can be very useful in the early phases of a project [13].

An estimation of required material quantities, concrete, and reinforcement in multistorey buildings was performed by Mučenski et al. The forecasting model was defined using neural networks. Model analysis and definition data were taken from 115 major multistorey projects. The input variables of the model for forecasting the required amount of concrete and reinforcement are as follows: total gross area, average gross floor area, number of stiffening walls, longitudinal raster, transverse raster, and type of landing structure. The best results were shown by a network trained with the BFGS (Broyden-Fletcher-Goldfarb-Shanno) algorithm with an average error of $12.49 \%$ [14].

Garcia de Sotto et al. made an estimate of the materials and presented the methodology which was used to achieve estimate of satisfactory accuracy in the early phases of project implementation. They used neural networks, among other things, for modelling. Obtained results showed a significant improvement compared to the situation in practice [15].

The same authors, Garcia de Sotto et al., aimed to devise a process for developing a model which would be used for the preparation of preliminary estimates of construction material quantities taking into consideration data which are available during the early phases of the project and to assess the model by using the Akaike information criterion. The 


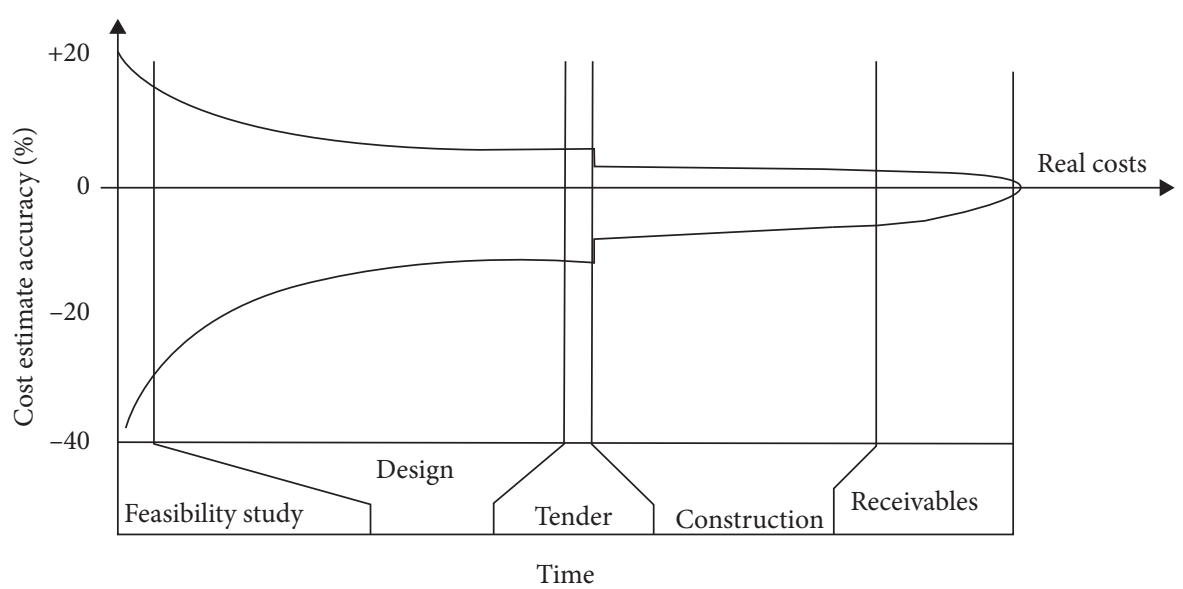

Figure 1: Cost estimate reliability [3].

proposed procedure is illustrated by an example in which data from 58 designs were used to define the model. These data were used for estimating used up concrete and reinforcement by using the neural network technique. For choosing the model with the highest accuracy, Akaike information criteria were used [16].

\section{Materials and Methods}

The first step undertaken for the purposes of this research is data collection and analysis. The data collected, after analysis, had to be prepared for model formation. In the end, two final models for the estimation of construction material consumption were defined, one for the concrete consumption and the other for reinforcement consumption.

The data were collected from the Main Designs of Integral Road Bridges, which were built on highways in the territories of Montenegro, Bosnia and Herzegovina, and Serbia. The term integral bridges is a modern term for concrete and composite frame structures of bridges without expansion joints and bearings [17]. There are several definitions of integral bridges. According to some, integral bridges are single-span frames with no expansion joints and bearings. In addition to this, we can find other definitions in the literature. They define this type of bridges as continuous frames without expansion joints and bearings just above the piers.

The research included 101 structures. Among them, there are 48 bridges from Montenegro, 29 bridges from Bosnia and Herzegovina, and 24 bridges from Serbia. The design documentation was prepared in three countries, so its form differs from one another. Therefore, the analysis and data preparation processes were complex. Only the same types of work were taken from all the bills of quantities and cost estimates in order to achieve data uniformity. In bills of quantities and cost estimates, the types of works are divided into preliminary, earthworks, concrete, reinforcement, tensioning works and prestressing, insulating, asphalt, and finishing works. Data on quantities of concrete and reinforcement were taken from concrete and reinforcement types of work.
An integral part of the technical documentation for the main design of bridges is the bill of quantities and cost estimate, and all the necessary data are obtained from them. The spans of these bridges range from 11.5 to 28 meters, the number of spans is from 1 to 18 , the length of bridges without wing walls ranges from 11.5 to 784.4 meters, and the pier height is from 2.8 up to 65 meters. These projects were carried out in the period from 2010 until 2016.

After collecting the material quantity data, model input data were determined. Model inputs will represent certain design characteristics of bridges. The criteria for choosing such characteristics were their direct impact on material consumption. Based on this, the following characteristics were chosen: bridge length, bridge width, pier height, and bridge span. The data about bridge characteristics were taken from the main designs of these structures. Some of the data, such as pier height and bridge span, had to be corrected in such a way that a single value could be used as an input size. This is the reason why, when pier height is mentioned, as input data, it implies the mean height of middle piers. In case of a single-span structure, as a mean pier height, it is implied that the mean abutment height was used. Regarding the span of the bridge, this parameter had to be corrected for the fact that it is not the same if you have a larger number of smaller spans or a smaller number of larger spans in the identical length of the bridge. Due to this fact, the bridge span, as an input parameter, implies the mean of the span. The input data, prepared in the aforementioned manner, are presented with their limit and mean values in the table (see Table 1).

In order to improve the model for the material consumption estimate, as the input parameters, the data on construction technology and structure foundation are introduced.

For the span structure of analysed bridges, two types of formworks were used: formworks on a fixed scaffolding and formworks on mobile scaffolding. For that reason, the new input variable, named construction technology, has a value of 0 for formworks on a fixed scaffolding and the value is 1 when the formworks are on a mobile scaffolding.

The method of founding determines the amount of material used for founding. The bridges whose data were 
TABle 1: Input data.

\begin{tabular}{|c|c|c|c|c|c|c|}
\hline Input data number & Input data description & Data type & Meas. unit & Min & Max & Mean value \\
\hline Input 1 & Bridge length & Numeric & $\mathrm{m}$ & 11.5 & 784.4 & 153.25 \\
\hline Input 2 & Bridge width & Numeric & $\mathrm{m}$ & 6.5 & 30.55 & 11.52 \\
\hline Input 3 & Pier height & Numeric & $\mathrm{m}$ & 2.8 & 35.9 & 13.65 \\
\hline Input 4 & Bridge span & Numeric & $\mathrm{m}$ & 11.3 & 44.5 & 24.07 \\
\hline Input 5 & Construction technology & Discrete & - & 0 & 1 & - \\
\hline Input 6 & Founding method & Discrete & - & 0 & 2 & - \\
\hline
\end{tabular}

used in this research were founded shallow, deep, or combined. The new input variable, named the founding method, depending on the method of founding, has the following values: 0 in case of shallow founding, 1 in case of deep founding, and 2 in case of combined founding.

The next step is defining the model output data. Based on the considered parts of the research, one output from each model was determined, which is the total amount of concrete and the total amount of reinforcement for the construction of integral road bridges (see Tables 2 and 3).

In the process of model formation using artificial neural networks, the available data should be divided into two sets. These two sets represent the training and test sets. The data of the training set are used for training and from the test set for checking the network.

Various recommendations can be found in the literature regarding the percentage ratio of these sets. A large number of authors select data in the ratio $90 \%$ to $10 \%, 80 \%$ to $20 \%$, $85 \%$ to $15 \%$, or $70 \%$ to $30 \%$ [18]. Of course, these are just recommendations, and the specificity of each of the problems being solved makes us decide on the appropriate ratio between the two sets. In this research, the training and test set will be divided in the ratio $80 \%$ to $20 \%$. In 6 models, a direct division will be made into training and test sets, and in 2 models, a random selection of data will be done. The crossvalidation procedure ( $k$-fold cross-validation and leave-oneout cross-validation) shall be used to randomly select data.

Network training is preceded by a transformation, i.e., scaling data to fit everyone within a certain size range. The choice of ranges for scaling inputs and outputs depends on the activation function of the output quantities. Data can be scaled using standardization and normalization $[19,20]$. The result of these methods is to reduce certain data to the same order of magnitude. Moreover, they enable the analysis of data of the same importance when forming the model, which means that it will also provide data analysis with a smaller size range. The data scaling methods used in the study are StandardScaler (Z-score normalization) and min-max normalization.

Network formation begins with determining the network architecture. This involves defining the number of layers and the number of neurons in each of the layers. Some authors recommend that it is not necessary to take more than two hidden layers when defining an artificial neural network $[18,21,22]$. The confirmation that the networks with two hidden layers gave reliable results is found in many theoretical results and numerous simulations in various engineering fields. In addition, there are theoretical results that indicate that a single hidden layer is sufficient for the network to approximate any complex nonlinear function with sufficient accuracy [23].

The number of neurons in the hidden layers is not uniquely determined. There are recommendations in the literature but not a precise and reliable way of determining them. A large number of neurons lead to the problem of overfitting, while the insufficient number of neurons leads to the problem of underfitting, i.e., poor approximation of the dependence between input and output quantities. The number of neurons should be such that it does not lead to any of these issues, but to enable data to exhibit its most useful characteristics. The recommendations made by some authors refer to the upper limit of the number of neurons in the hidden layer. Lippmann (1987), Nielsen (1987), and Hecht-Nielsen (1990) recommend determining the number of neurons following inequality (1), whereas Rogers and Dowla (1994) give recommendations for the maximum number of neurons, $N_{\mathrm{H}}$, following inequality (2). It is advisable to accept a smaller number from the ones stated in the inequality, where $N_{\mathrm{i}}$ is the number of input parameters and $N_{\mathrm{S}}$ is the number of training samples:

$$
\begin{aligned}
& N_{\mathrm{H}} \leq 2 \times N_{\mathrm{i}}+1, \\
& N_{\mathrm{H}} \leq \frac{N_{\mathrm{S}}}{N_{\mathrm{i}}+1} .
\end{aligned}
$$

In the process of defining a model, one must strive to find a model with the best possible opportunity for generalization. Generalization is a process in which knowledge that is valid for a certain set of cases is transferred to some of its supersets [24], i.e., based on data which are not presented to the model during the training (the validation set), the model has the ability to result in satisfactory sizes even though based on data which are not presented during training. The validation set is introduced to avoid the problem of overfitting or determine stopping points of the training process [25]. Generalization in forecasting is further enhanced by the cross-validation process. This procedure is performed on the data from the test set.

Constant performance measurement is done during the model definition. Performance measurement, in fact, is an accuracy forecast. The difference between the actual (desired) and the forecast value is the forecasting error, and a measure of accuracy is defined. There are a number of accuracy measures for forecasting in the literature. The accuracy of the model in this study was determined using the mean absolute percentage error (MAPE). A satisfactory generalization probability in models is achieved if the 
TABle 2: Output data of the first model.

\begin{tabular}{lcccccc}
\hline Output data number & Output data description & Data type & Meas. unit & Min & Max & Mean value \\
\hline Output 1 & Total quantity of concrete & Numeric & $\mathrm{m}^{3} / \mathrm{m}^{2}$ & 1.05 & 3.11 & 1.54 \\
\hline
\end{tabular}

TABle 3: Output data of the second model.

\begin{tabular}{|c|c|c|c|c|c|c|}
\hline Output data number & Output data description & Data type & Meas. unit & Min & Max & Mean value \\
\hline Output 1 & Total quantity of reinforcement & Numeric & $\mathrm{kg} / \mathrm{m}^{2}$ & 117.26 & 415.58 & 250.8 \\
\hline
\end{tabular}

TABLE 4: Activation functions of a multilayer perceptron model of the artificial neural network.

\begin{tabular}{|c|c|c|c|}
\hline Function & Mark & Explanation & Range \\
\hline Identity & $x$ & Only in the output layer & $(-\infty,+\infty)$ \\
\hline $\begin{array}{l}\text { Rectified linear unit } \\
\text { function }\end{array}$ & $\operatorname{Max}(0, x)$ & $\begin{array}{l}\text { Neuron activation is forwarded directly as an output if positive, and if negative, it is } \\
\text { forwarded to 0. It has been shown to have } 6 \text { times better convergence than a } \\
\text { hyperbolic tangent function }\end{array}$ & $(0,+\infty)$ \\
\hline A hyperbolic tangent & {$\left[2 /\left(1+e^{-2 x}\right)\right]-1$} & $\begin{array}{c}\text { Neuron activation is forwarded directly as an output if positive, and if negative, it is } \\
\text { forwarded to } 0 . \text { It has been shown to have } 6 \text { times better convergence than a } \\
\text { hyperbolic tangent function }\end{array}$ & $(-1,+1)$ \\
\hline
\end{tabular}

deviation between the forecasted and expected results at the training and test set is small.

The forecasting model was formed in Python 3.7 software package. In order to solve the problem that is the subject of the research, models about estimating material consumption, a multilayer perceptron MLP is formed, which is one of the artificial neural network types.

The most commonly used neuron activation functions in the hidden layers are logistic sigmoid (logistic), a hyperbolic tangent (tanh), and the function of rectified linear unit (ReLu). The activation function of output neurons is mostly linear. Bearing in mind, the number and other data characteristics, following the aforementioned recommendations, during the model formation, for hidden neurons, the function of rectified linear unit (ReLu), and a hyperbolic tangent (tanh) were used, whereas for the output neurons, the identity function was used (see Table 4).

\section{Results}

Artificial neural network models, multilayer perceptron (MLP), are formed based on defined input and output sizes and other required parameters. The number of layers as well as the number of neurons in hidden layers is determined based on recommendations, and the number of neurons in the input and output layer is determined based on the number of input and output sizes. The largest number of hidden neurons which was taken in the models is 13 based on expressions (1) and (2). 8 artificial neural network models were formed. In one half of these models, the data were used which were scaled by using the StandardScaler procedure, whereas for the other the minmax procedure was used.

All neural networks in both models, NMB1, NMB2, NMB3, NMB4, NMB5, NMB6, NMB7, and NMB8 for the model forecasting concrete consumption and NMA1, NMA2, NMA3, NMA4, NMA5, NMA6, NMA7, and NMA8 for the model forecasting reinforcement consumption, have 6 input and 1 output size. Neural network models with StandardScaler standardization for forecasting concrete consumption and reinforcement consumption are presented in tables (see Tables 5 and 6). They also list the characteristics of each model with a measure of accuracy given by the mean absolute percentage error (MAPE).

The following three neural network models are formed using the data which were scaled by applying the principle of min-max normalization. Data about model characteristics as well as the estimation accuracy which is determined by the mean absolute percentage error (MAPE) are presented in tables (see Tables 7 and 8).

Random data selection was done with two models using $\mathrm{k}$-fold cross-validation for $k=10$ and leave-one-out crossvalidation (LOOCV). Estimation accuracy in these models is determined through mean absolute percentage error (MAPE) (see Tables 9-12). In those models where data division was done in accordance with $k$-fold cross-validation, the data were scaled by using a Standard Scaler, and in those models where the division was done with LOOCV, the data were scaled with min-max function. Two of each model that gave the best results are presented here.

By comparing presented models, it can clearly be seen that models NMB1 and NMA8 have the highest estimation accuracy. For model NMB1, StandardScaler was used for scaling the data. It defines 3 layers of neurons, one of which is input and one output layer. In the hidden layer, there are 12 neurons. The activation function of a hidden layer is the function of rectified linear unit (ReLu). The measure of the accuracy assessment model is expressed through mean absolute percentage error and is $8.56 \%$.

Model NMA 8 processed data which were scaled by using min-max normalization. The network architecture of this model is represented by 3 layers of neurons. There are 6 neurons in the input layer, 1 in the output, and 9 neurons in a hidden layer. The activation function of a hidden layer is 
TABLE 5: Artificial neural network models for estimating concrete consumption (StandardScaler).

\begin{tabular}{lccccc}
\hline $\begin{array}{l}\text { Model } \\
\text { name }\end{array}$ & $\begin{array}{c}\text { Model } \\
\text { characteristics }\end{array}$ & $\begin{array}{c}\text { Activation function of hidden } \\
\text { layers }\end{array}$ & $\begin{array}{c}\text { Activation function of an } \\
\text { output layer }\end{array}$ & $\begin{array}{c}\text { MAPE training set } \\
(\%)\end{array}$ & $\begin{array}{c}\text { MAPE test set } \\
(\%)\end{array}$ \\
\hline NMB1 & MLP 6-12-1 & ReLu & Identity & 7.68 & 8.56 \\
NMB2 & MLP 6-4-1 & Tanh & Identity & 10.7 & 11.51 \\
NMB5 & MLP 6-7-1 & ReLu & Identity & 8.01 & 9.95 \\
\hline
\end{tabular}

TABLe 6: Artificial neural network models for estimating reinforcement consumption (StandardScaler).

\begin{tabular}{lcccc}
\hline $\begin{array}{l}\text { Model } \\
\text { name }\end{array}$ & $\begin{array}{c}\text { Model } \\
\text { characteristics }\end{array}$ & $\begin{array}{c}\text { Activation function of hidden } \\
\text { layers }\end{array}$ & $\begin{array}{c}\text { Activation function of an } \\
\text { output layer }\end{array}$ & $\begin{array}{c}\text { MAPE training set } \\
(\%)\end{array}$ \\
\hline NMA1 & MLP 6-7-1 & Tanh & Identity & 18.85 \\
NMA2 & MLP 6-13-1 & ReLu & Identity & 10.83 \\
NMA5 & MLP 6-8-1 & ReLu & Identity & 16.74 \\
\hline
\end{tabular}

TABLE 7: Artificial neural network models for estimating concrete consumption (min-max normalization).

\begin{tabular}{lccccc}
\hline $\begin{array}{l}\text { Model } \\
\text { name }\end{array}$ & $\begin{array}{c}\text { Model } \\
\text { characteristics }\end{array}$ & $\begin{array}{c}\text { Activation function of hidden } \\
\text { layers }\end{array}$ & $\begin{array}{c}\text { Activation function of an } \\
\text { output layer }\end{array}$ & $\begin{array}{c}\text { MAPE training set } \\
(\%)\end{array}$ & $\begin{array}{c}\text { MAPE test set } \\
(\%)\end{array}$ \\
\hline NMB3 & MLP 6-11-1 & ReLu & Identity & 9.97 & 10.5 \\
NMB4 & MLP 6-12-1 & Tanh & Identity & 10.78 \\
NMB6 & MLP 6-7-1 & ReLu & Identity & 8.78 & 10.81 \\
\hline
\end{tabular}

TABLE 8: Artificial neural network models for estimating reinforcement consumption (min-max normalization).

\begin{tabular}{|c|c|c|c|c|c|}
\hline $\begin{array}{l}\text { Model } \\
\text { name }\end{array}$ & $\begin{array}{c}\text { Model } \\
\text { characteristics } \\
\end{array}$ & $\begin{array}{l}\text { Activation function of hidden } \\
\text { layers }\end{array}$ & $\begin{array}{l}\text { Activation function of an } \\
\text { output layer }\end{array}$ & $\begin{array}{c}\text { MAPE training set } \\
(\%)\end{array}$ & $\begin{array}{l}\text { MAPE test set } \\
(\%)\end{array}$ \\
\hline NMA3 & MLP 6-7-1 & $\mathrm{ReLu}$ & Identity & 18.28 & 19.03 \\
\hline NMA4 & MLP 6-3-1 & Tanh & Identity & 19.15 & 19.41 \\
\hline NMA6 & MLP 6-9-1 & ReLu & Identity & 18.26 & 18.78 \\
\hline
\end{tabular}

TABLE 9: Artificial neural network models with random data choice for estimating concrete consumption ( $k$-fold cross-validation, $k=10)$.

\begin{tabular}{lccccc}
\hline $\begin{array}{l}\text { Model } \\
\text { name }\end{array}$ & $\begin{array}{c}\text { Data-scaling } \\
\text { procedure }\end{array}$ & $\begin{array}{c}\text { Model } \\
\text { characteristics }\end{array}$ & $\begin{array}{c}\text { Activation function of } \\
\text { hidden layers }\end{array}$ & $\begin{array}{c}\text { Activation function of an } \\
\text { output layer }\end{array}$ & $\begin{array}{c}\text { MAPE } \\
(\%)\end{array}$ \\
\hline NMB7 & StandardScaler & MLP 6-4-1 & $\begin{array}{c}\sigma \\
(\%)\end{array}$ \\
\hline
\end{tabular}

TABLE 10: Artificial neural network models with random data choice for estimating concrete consumption (LOOCV).

\begin{tabular}{|c|c|c|c|c|c|c|}
\hline $\begin{array}{l}\text { Model } \\
\text { name }\end{array}$ & $\begin{array}{l}\text { Data-scaling } \\
\text { procedure }\end{array}$ & $\begin{array}{c}\text { Model } \\
\text { characteristics } \\
\end{array}$ & $\begin{array}{c}\text { Activation function of } \\
\text { hidden layers }\end{array}$ & $\begin{array}{c}\text { Activation function of an } \\
\text { output layer }\end{array}$ & $\begin{array}{l}\text { MAPE training } \\
\text { set }(\%)\end{array}$ & $\begin{array}{c}\text { MAPE test set } \\
(\%)\end{array}$ \\
\hline NMB8 & Min-max & MLP 6-11-1 & ReLu & Identity & 10.69 & 11.06 \\
\hline
\end{tabular}

TABLE 11: Artificial neural network models with random data choice for estimating reinforcement consumption ( $k$-fold cross-validation, $k=10)$.

\begin{tabular}{|c|c|c|c|c|c|c|}
\hline $\begin{array}{l}\text { Model } \\
\text { name }\end{array}$ & $\begin{array}{c}\text { Data-scaling } \\
\text { procedure }\end{array}$ & $\begin{array}{c}\text { Model } \\
\text { characteristics }\end{array}$ & $\begin{array}{l}\text { Activation function of hidden } \\
\text { layers }\end{array}$ & $\begin{array}{c}\text { Activation function of an } \\
\text { output layer }\end{array}$ & $\begin{array}{c}\text { MAPE } \\
(\%) \\
\end{array}$ & $\begin{array}{c}\sigma \\
(\%) \\
\end{array}$ \\
\hline NMA7 & StandardScaler & MLP 6-4-1 & $\mathrm{ReLu}$ & Identity & 22.91 & 2.5 \\
\hline
\end{tabular}

TABLE 12: Artificial neural network models with random data choice for estimating reinforcement consumption (LOOCV).

\begin{tabular}{|c|c|c|c|c|c|c|}
\hline $\begin{array}{l}\text { Model } \\
\text { name }\end{array}$ & $\begin{array}{l}\text { Data scaling } \\
\text { procedure }\end{array}$ & $\begin{array}{c}\text { Model } \\
\text { characteristics }\end{array}$ & $\begin{array}{c}\text { Activation function of } \\
\text { hidden layers }\end{array}$ & $\begin{array}{c}\text { Activation function of an } \\
\text { output layer }\end{array}$ & $\begin{array}{c}\text { MAPE training } \\
\text { set }(\%)\end{array}$ & $\begin{array}{l}\text { MAPE test set } \\
(\%)\end{array}$ \\
\hline NMA8 & Min-max & MLP 6-9-1 & ReLu & Identity & 14.12 & 17.31 \\
\hline
\end{tabular}


the function of a rectified linear unit (ReLu). Mean absolute percentage error is $17.31 \%$.

The two models with the highest accuracy were selected as the final models for the estimation of concrete and reinforcement consumption, and based on them, the forecasting models were defined.

\section{Conclusion}

Based on the results presented in the study, it is concluded that the models with the highest accuracy of concrete consumption and reinforcement for the construction of integral road bridges are artificial neural network models whose architecture is represented by three layers of neurons, six of which are in the first layer, and one in the last output layer. In the hidden layer, there are 12 neurons in the concrete consumption estimation model, while 9 neurons are in the reinforcement consumption estimation model. The activation function of the hidden layers of neurons is the function of a rectified linear unit (ReLu), while the activation function of the output layers is linear (Identity). The accuracy measure is represented in both models by mean absolute percentage error (MAPE). In the model for concrete consumption, $\mathrm{MAPE}=8.56 \%$, whereas $\mathrm{MAPE}$ for the estimate of reinforcement consumption is $17.31 \%$.

It is possible to improve the accuracy of a forecasting model by increasing the number of data in the data base. Additionally, the forecasting model would have the potential to be more widely applied if the database was expanded with certain features of the structures such as the type of cross section, height of the cross section, number of spans, number of piers, and structural system. The database could be improved by entering data on the category of the road (type and significance of the road) on which the bridges are located. The justification for the existence of this type of data in the database lies in the fact that the category of the road directly affects the load of bridges, which affects main characteristics of bridges and thus the amount of concrete and reinforcement. The potential parameters by which the database could be expanded would, in fact, be the input parameters of the forecasting model.

The use of a forecasting model would be particularly beneficial to the contractor when he is also the designer (for the contract-type design-build). With the help of a forecast model, without having to develop the preliminary design and only on the basis of sketches, the contractor could estimate the amount of material. The estimated amount of material is significant to him in the competitive bidding phase in order to submit as precise a bid as possible.

In the early phases, the amount of data available on future structures is insufficient for forming the accurate estimate. This means that the error in estimating material consumption is also greater than the estimates made in the subsequent phases of implementation. Quantity, type, and quality of data, which are available at the time of evaluation, condition the application of a model. This is the reason why it is necessary to adjust the input parameters to the data we have. The estimation importance in the early phases lies in the fact that the results of this early estimate directly affect assessing a total cost which in the further process determines/recommends us, or not for entering into the project implementation.

\section{Data Availability}

The data used to support the findings of this study are available from the corresponding author upon request.

\section{Conflicts of Interest}

The authors declare that there are no conflicts of interest regarding the publication of this paper.

\section{References}

[1] Institute for Project Management, Guide through the Corpus of Knowledge for Project Management, FTS, Novi Sad, Serbia, 2010, in Serbian.

[2] J. E. Rowings, The Civil Engineering Handbook: Construction Estimating, CRC Press, Taylor and Francis Group, Boca Raton, FL, USA, 2nd edition, 2003.

[3] P. S. Brandon, Quantity Surveying Techniques-New Directions, Blackwell Scientific Publications, Hoboken, NJ, USA, 1992.

[4] H. Adeli and C. Yeh, "Perceptron learning in engineering design," Microcomputers in Civil Engineering, vol. 4, no. 4, pp. 247-256, 1989.

[5] J. Sodikov, "Cost estimation of highway projects in developing countries: artificial neural network approach," Journal of the Eastern Asia Society for Transportation Studies, vol. 6, pp. 1036-1047, 2005.

[6] X. Wang, X. Duan, and J. Liu, "Application of neural network in the cost estimation of highway engineering," Journal of computers, vol. 5, no. 11, 2010.

[7] C. G. Wilmont and G. Cheng, "Estimating future highway construction costs," Journal of Construction Engineering and Management, vol. 129, no. 3, 2003.

[8] C. G. Wilmont and B. Mei, "Neural network modeling of highway construction costs," Journal of Construction Engineering and Management, vol. 131, no. 7, 2005.

[9] H. M. Gunaydin and S. Z. Dogan, "A neural network approach for early cost estimation of structural systems of buildings," International Journal of Project Management, vol. 22, no. 7, pp. 595-602, 2004.

[10] M. B. Kazez and C. Vipulanandan, "Bridge damage and repair cost estimates after a hurricane," in Proceedings of the THC 2010 Conference \& Exhibition, Houston, TX, USA, 2010.

[11] E. Atta-Asiamah, "Estimation of the cost of building a water treatment plant and related facilities for Kaw city," Thesis, Faculty of the Graduate College of the Oklahoma State University, Kaw, OK, USA, 2005.

[12] T. K. Burrows, I. Pegg, and J. Martin, "Predicting building construction duration," ACCE International Transactions PS, vol. 14, 2005.

[13] N. Fragkakis, S. Lambropoulos, and G. Tsiambaos, "Parametric model for conceptual cost estimation of concrete bridge foundations," Journal of Infrastructure Systems Volume, vol. 17, no. 2, 2011.

[14] V. Mučenski, I. Pesko, M. Trivunic, J. Drazic, and G. Cirovic, "Neural network optimization for estimating the required quantities of concrete and reinforcement in multi-storey buildings," Building Materials and Constructions, vol. 55, no. 2, pp. 27-46, 2012, in Serbian. 
[15] B. Garcia de Sotto, B. T. Adey, and D. Fernando, "A hybrid methodology to estimate construction material quantities at an early project phase," International Journal of Construction Management, vol. 17, no. 3, pp. 165-196, 2017.

[16] B. Garcia de Sotto, B. T. Adey, and D. Fernando, "A process for the development and evaluation of preliminary construction material quantity estimation models using backward elimination regression and neural networks," Journal of Cost Analysis and Parametrics, vol. 7, no. 3, pp. 180-218, 2014.

[17] M. Przulj, Bridges, Association "Izgradnja", Belgrade, Serbia, 2014, in Serbian.

[18] G. Zhang, B. E. Patuwo, and M. Y. Hu, Forecasting with Artificial Neural networks:The State of the Art, Graduate School of Management, Kent State University, Kent, $\mathrm{OH}$, USA, 1997.

[19] J. Brownlee, Machine Learning Mastery with Weka, Machine Learning Mastery Pty. Ltd., Australia, 2016.

[20] Z. Beljkas and M. Knezevic, Estimation of the Cost of Integral Bridges Using Artificial Intelligence, Journal of the Croation Association of Civil Engineers, Zagreb, Croatia, 2020.

[21] I. Goodfelow, Y. Bengio, and A. Courville, Deep Learning (Adaptive Computation and Machine Learning Series), The MIT Press, Cambridge, England, 2016.

[22] R. Reed and R. Marksll, Neural Smithing: Supervised Learning in Feedforward Artificial Neural Networks (A Bradford Book), The MIT Press, Cambridge, UK, 1999.

[23] G. Cybenko, "Approximation by superpositions of a sigmoidal function," Mathematics of Control, Signals, and Systems, vol. 2, no. 4, pp. 303-314, 1989.

[24] P. Janicic and M. Nikolic, Artificial Intelligence, Faculty of Mathematics, University of Belgrade, Belgrade, Serbia, 2019, in Serbian.

[25] M. Simonovic, "Application of artificial neural networks for short-term prediction and analysis of district heating systems," Doctoral Dissertation, Faculty of Mechanical Engineering, University of Nis, Nis, Serbia, 2016. 